\title{
Well-Conditioned Asymptotic Waveform Evaluation for Efficient Computation of Wave-Scattering from Perfectly Conducting Bodies
}

\author{
Patrick Bradley, Conor Brennan and Marissa Condon, \\ RF modelling and simulation group, RINCE, School of Electronic Engineering, Dublin City University, \\ Dublin, Ireland, Telephone: (353) 17005856, Email: bradleyp@eeng.dcu.ie
}

\begin{abstract}
This paper presents a model order reduction algorithm for the surface electric field integral equation (EFIE) formulation of the electromagnetic wave scattering problem. The method allows fast and accurate frequency sweep calculations of electromagnetic wave scattering from a perfectly conducting (PEC) three-dimensional object. We apply the Well-Conditioned Asymptotic Waveform Evaluation (WCAWE) method to circumvent the computational complexity associated with the numerical solution of such formulations. Practical implementation issues are addressed with numerical examples given to illustrate the accuracy and robustness of the proposed methods.
\end{abstract}

\section{INTRODUCTION}

The surface electric field integral equation in conjunction with the method of moments (MoM) is a popular frequency domain technique for analysis of electromagnetic scattering from homogenous bodies of arbitrary shape [1], [2]. Many problems, such as the evaluation of radar cross section (RCS), require the determination of the system response at multiple frequencies. For such analysis, the surface current density needs to be calculated at many frequencies. This can be very time consuming when using traditional frequency domain numerical methods, based on the solution of a dense matrix equation, at each frequency [3]. Developments in computational iterative techniques which incorporate fast algorithms can alleviate this problem. Acceleration techniques include the Conjugate Gradient Fast Fourier Transform method (CG-FFT), the Fast Multipole Method (FFM) [1] and the precorrected FFT methods.

To alleviate the computational burden of multiple simulations, several alternative approaches have been proposed [3] [5]. Many of these focus on using a rational function to approximate the solution vector and its derivatives at a central frequency and subsequently exploiting this information to reconstruct the solution within the frequency band of interest. This is possible because coefficients of the system matrix equation, describing the system behaviour, are known functions of frequency. The low order of the approximate system matrix permits its inverse to be computed with negligible computational effort. Hence, once the reduced order system has been constructed the frequency response can be evaluated at an almost arbitrary number of frequency samples, resulting in a significant reduction in computational cost. Padé via Asymptotic Waveform Evaluation (AWE) [3], [6], Passive Reduced-order Interconnect macro Modeling Algorithm
(PRIMA) and other model order reduction techniques such as Padé via Lanczos (PVL) [4] have been used for efficient solution of a wide variety of linear problems. Extending PVL and PRIMA to handle systems with nonlinear parameters, such as frequency dependence can prove prohibitive for large scale computations. The AWE method, originally developed for circuit analysis, is a moment matching technique that expands the solution vector in terms of a Taylor series. The solution for each unknown and its derivatives are calculated and used to form the Padé rational function approximation. This in turn is used to improve the radius of convergence of the Taylor series in order to reconstruct the solution over a wider frequency band. However, algorithms such as the Padé via AWE that are based on explicit moment matching of the frequency response exhibit certain numerical stability problems and are inherently ill-conditioned, inevitably leading to stagnation in the moment matching process [3], [6].

In this paper an efficient and numerical stable projection based algorithm for model order reduction, called the WellConditioned Asymptotic Waveform Evaluation (WCAWE) and originally proposed in [5], is applied to the surface integral equation formulation. This algorithm produces a wellconditioned and high-order approximation from a single expansion point with a significantly wider bandwidth than that obtained from Padé via AWE and its variants. To the knowledge of the authors, the WCAWE technique has not been previously applied to a MoM solution of the surface IE formulation. It is the purpose of this paper to investigate the use of the WCAWE technique to obtain a fast frequency sweep solution for the current density in a PEC three-dimensional structure.

\section{SURFACE ELECTRIC FIELD INTEGRAL EQUATION FORMULATION}

The work presented in this paper examines scattering from a three dimensional perfectly conducting object. A time dependence of $\exp (\jmath \omega t)$ is assumed and suppressed. The object is illuminated by a plane wave and the scattered electric field $\mathbf{E}^{s}$ can be computed from the surface current by the following electric field integral equation [1], [2], [6] which applies to points on the surface of the body.

$$
\mathbf{E}_{t a n}^{i}=-\mathbf{E}_{t a n}^{s}
$$


where $\mathbf{E}^{s}(\mathbf{r})=-\jmath \omega \mathbf{A}-\nabla \phi$ and $\mathbf{A}, \phi$ are the magnetic vector and scalar potential respectively. To solve the surface EFIE numerically the method of moments is applied. The conducting surface $S$ is discretised and the current on the surface is expanded using the Rao-Wilton-Glisson (RWG) basis function $\mathbf{f}_{n}(\mathbf{r})[2]:$

$$
\mathbf{J}(k, \mathbf{r})=\sum_{n=1}^{m} I_{n}(k) \mathbf{f}_{n}(\mathbf{r})
$$

where $m$ is the number of unknowns. Applying a testing procedure to Equation 1 leads to the following matrix equation:

$$
\mathbf{Z}(k) \mathbf{j}(k)=\mathbf{v}(k)
$$

where $\mathbf{v}(k)$ is the incident field vector and $\mathbf{Z}(k)$ is a $m \times$ $m$ matrix containing coupling information between the basis functions. The element in the $m^{\text {th }}$ row and $n^{\text {th }}$ column of $\mathbf{Z}(k)$ is given by [2], [6]:

$$
\begin{aligned}
& Z_{m n}(k)=\jmath k \eta_{0} \iint \mathbf{f}_{m}(\mathbf{r}) \cdot \iint \mathbf{f}_{n}\left(\mathbf{r}^{\prime}\right) g\left(\mathbf{r}, \mathbf{r}^{\prime}\right) d \mathbf{r}^{\prime} d \mathbf{r} \\
& -\frac{\jmath \eta_{0}}{k} \iint \nabla \cdot \mathbf{f}_{m}(\mathbf{r}) \cdot \iint\left(\nabla^{\prime} \cdot \mathbf{f}_{n}\left(\mathbf{r}^{\prime}\right)\right) g\left(\mathbf{r}, \mathbf{r}^{\prime}\right) d \mathbf{r}^{\prime} d \mathbf{r}
\end{aligned}
$$

where $g\left(\mathbf{r}, \mathbf{r}^{\prime}\right)$ denotes the Green's function, given by:

$$
g\left(\mathbf{r}, \mathbf{r}^{\prime}\right)=\frac{e^{-\jmath k\left|\mathbf{r}-\mathbf{r}^{\prime}\right|}}{4 \pi\left|\mathbf{r}-\mathbf{r}^{\prime}\right|} .
$$

Similarly $V_{m}(k)=\iint \mathbf{f}_{m}(\mathbf{r}) \cdot \mathbf{E}_{i n c}(k) d \mathbf{r}$, where $\mathbf{E}_{i n c}$ denotes the plane wave incident field [2], [6]. $\mathbf{k}$ represents the propagation wave vector of the incident field. Equation 3 can be solved for $\mathbf{j}(k)$ to determine the total unknown current $\mathbf{J}(k, \mathbf{r})$ throughout the scatterer. Once $\mathbf{j}(k)$ is obtained, other quantities, such as the RCS can be computed [1], [6].

\section{REVIEW OF ASYMPTOTIC WAVEFORM EVALUATION VIA PADÉ APPROXIMATION}

The central goal in AWE is to determine the system behaviour over a wide frequency range from the solution at one or several frequency points [3], [6], [7]. This is possible because coefficients of the matrix equation, describing the system behaviour, are known functions of frequencies. The AWE method approximates the frequency response by expanding $\mathbf{j}(k)$ in a Taylor series around $k_{0}$ :

$$
\mathbf{j}(k)=\sum_{n=0}^{\mathcal{O}} \mathbf{m}_{n}(\xi \sigma)^{n}
$$

where $\mathbf{m}_{n}$ are the moments of $\mathbf{j}(k), \mathcal{O}$ is the order of the Taylor series expansion and $\sigma=\left(k-k_{0}\right)$, where $k_{0}$ is the wave number at the expansion frequency. By substituting Equation 6 into Equation 3, expanding the impedance matrix $\mathbf{Z}(k)$ and the excitation vector $\mathbf{v}(k)$ in a Taylor series, we can re-write Equation 3 as:

$$
\sum_{n=0}^{\mathcal{O}}\left(\mathbf{Z}_{n} \sigma^{n}\right) \mathbf{j}(k)=\sum_{n=0}^{\mathcal{O}}\left(\mathbf{v}_{n} \sigma^{n}\right)
$$

Finally, equating the coefficients of equal powers of $\sigma$ on both sides of Equation 7 yields the recursive relation for the moment vectors:

$$
\mathbf{m}_{n}=\mathbf{Z}^{-1}\left(k_{0}\right)\left[\frac{\mathbf{v}_{n}\left(k_{0}\right)}{n ! \xi^{n}}-\sum_{q=0}^{n} \frac{\left(1-\delta_{q 0}\right) \mathbf{Z}_{q}\left(k_{0}\right) \mathbf{m}_{n-q}}{q ! \xi^{q}}\right]
$$

where $\delta_{q 0}$ is the Kronecker delta and $\xi$ is a scaling factor used to improve the conditioning of the Pade coefficient matrix $\mathbf{H}_{n}$ [3]. This scaling factor is chosen such that all the moments $\mathbf{m}_{n}$ are of the same order of magnitude and is given by:

$$
\xi=\frac{\left\|\mathbf{m}_{1}\right\|_{2}}{\left\|\mathbf{m}_{0}\right\|_{2}} .
$$

Vector $\mathbf{v}_{q}\left(k_{0}\right)$ denotes the $q^{\text {th }}$ derivative with respect to $k_{0}$ of $\mathbf{v}(k)$ evaluated at $k_{0}$. Similarly, $\mathbf{Z}_{q}\left(k_{0}\right)$ denotes the $q^{\text {th }}$ derivative of $\mathbf{Z}(k)$ evaluated at $k_{0}$. For the sake of clarity, $\mathbf{Z}_{q}\left(k_{0}\right), \mathbf{v}_{q}\left(k_{0}\right)$ and $\mathbf{j}(k)$ will be denoted by $\mathbf{Z}_{q}, \mathbf{v}_{q}$ and $\mathbf{j}$ respectively. Once the moment vectors are obtained, the value of $\mathbf{j}(k)$ at other frequencies can be calculated using Equation 6. However, the use of this expansion is limited to the radius of convergence of the Taylor series. In such cases, the rational function approach is used to improve the accuracy of the numerical solution. The Padé representations have a larger radius of convergence and therefore can provide a broader extrapolation as it includes poles as well as zeros in the response [3]. The aforementioned Padé approximations are given by:

$$
\sum_{n=0}^{L+M+1} \mathbf{m}_{n}(\xi \sigma)^{n}=\frac{\sum_{i=0}^{L} \mathbf{a}_{i}(\xi \sigma)^{i}}{1+\sum_{j=0}^{M} \mathbf{b}_{j}(\xi \sigma)^{j}}
$$

where the unknown coefficients $\mathbf{a}_{i}$ and $\mathbf{b}_{j}$ can be determined from $\mathbf{m}_{n}$ by multiplying Equation 10 by the denominator of the Padé expression. Matching the coefficients of the equals powers of $\sigma$ leads to a matrix equation containing the coefficient matrix $\mathbf{H}_{n}$, that allows for the solution of $\mathbf{b}_{j}$ to be obtained [3]. Having found the $\mathbf{b}_{j}$, the unknown coefficients $\mathbf{a}_{i}$ can be calculated as:

$$
\mathbf{a}_{i}=\mathbf{m}_{i}+\sum_{j=1}^{i} \mathbf{b}_{j} \mathbf{m}_{i-j} .
$$

\section{A. Numerical Instability}

The AWE moment matching subspace, $\mathbf{M}_{n}$, generated from the recursive Equation 8, is given by [8]:

$$
\mathbf{M}_{n}=\left[\mathbf{m}_{1}, \mathbf{m}_{2}, \ldots, \mathbf{m}_{n}\right]
$$

where $\mathrm{m}_{\mathrm{n}}$ are the moment vectors. It has been well documented [3], [7], [8] that the process of sequentially evaluating $\mathbf{m}_{n}$ is inherently ill-conditioned leading to instability in the computation of the Padé approximation. In a direct implementation, finite precision arithmetic causes each newly created moment vector $\mathbf{m}_{n}$ to converge towards the eigenvectors that are associated with the dominant eigenvalue of $\mathbf{Z}_{0}\left(k_{0}\right)$. As a result, the moments $\mathbf{m}_{n}$ contains only information 
corresponding to one eigenvalue of $\mathbf{Z}_{0}\left(k_{0}\right)$, even for small value of $n$ and as a consequence the moment matrix $\mathbf{H}_{n}$ becomes rapidly ill-conditioned. Subsequently, the solution of Equation 10 becomes unattainable, thus restricting its use to approximations of relatively low order, typically for values of $n<20$. It is hence, necessary to implement the construction of the $\mathbf{M}_{n}$ bases in a numerically stable way. This is generally done with the help of an orthogonalization process, whereby imposing an orthogonality relation among the vectors, linear independence can be maintained. Consequently, high order approximations can be constructed.

\section{WELL-CONDITIONED ASYMPTOTIC WAVEFORM EVALUATION}

A proposed approach that avoids the unattractive property of ill-conditioning of the AWE is the WCAWE [5], [7], [8], which introduces correction factors that eliminate illconditioning in order to obtain a high order approximation in a numerically stable manner. Orthogonalised Krylov subspace methods such as the Galerkin Asymptotic Waveform Evaluation (GAWE) [5], can also construct an arbitrarily high order stable approximation but in some cases will not match moments [5]. The WCAWE process, outlined in Appendix (1), rectifies this issue by the introduction of correction terms in the orthogonalisation process. The columns of $\mathbf{M}_{n}$ from Equation 12 are constructed iteratively by utilizing a modified Gram-Schmidt process. This procedure is used to orthogonalise $\mathbf{m}_{n}$ onto the basis:

$$
\widetilde{\mathbf{M}}_{n-1}=\left[\tilde{\mathbf{m}}_{1}, \tilde{\mathbf{m}}_{2}, \ldots, \tilde{\mathbf{m}}_{n-1}\right] .
$$

which is achieved by computing the orthogonal projection of $\tilde{\mathbf{m}}_{n}$ onto $\operatorname{span}\left\{\mathbf{m}_{1} \mathbf{m}_{2} \cdots \mathbf{m}_{n-1}\right\}$. This projection is subtracted from the original vector and the result is normalised to obtain $\mathbf{m}_{n}$. This is by construction, orthogonal to all previously computed vectors $\tilde{\mathbf{m}}_{1}, \tilde{\mathbf{m}}_{2}, \ldots, \tilde{\mathbf{m}}_{n-1}$ with unit norm. In this way, the orthogonality of the basis vectors is guaranteed and the moment matching process can be maintained. The resultant vector generated in Appendix (1) is given by [5], [7], [8]:

$$
\begin{aligned}
\tilde{\mathbf{m}}_{n}=\mathbf{Z}_{0}^{-1} & \left(\sum_{m=1}^{n-1}\left(\mathbf{v}_{m} \mathbf{e}_{1}^{T} \mathbf{P}_{\mathrm{U} 1}(n, m) \mathbf{e}_{n-m}\right)-\mathbf{Z}_{1} \mathbf{m}_{n-1}\right. \\
& \left.-\sum_{m=2}^{n-1} \mathbf{Z}_{m} \mathbf{M}_{n-m} \mathbf{P}_{\mathrm{U} 2}(n, m) \mathbf{e}_{n-m}\right)
\end{aligned}
$$

where $\mathbf{e}_{r}$ is the vector with the $r^{t h}$ entry equal to unity. $\widetilde{\mathbf{M}}_{n}$ and $\mathbf{M}_{n}$ are related by an $n \times n$ upper triangular, nonsingular matrix $\mathbf{U}$ created by the coefficients of the Gram-Schmidt process, and given by:

$$
\mathbf{M}_{n}=\widetilde{\mathbf{M}}_{n} \mathbf{U}^{-1}
$$

The correction term in Equation 14 is given by [5], [7], [8]:

$$
\mathbf{P}_{\mathrm{U} w}(n, m)=\prod_{t=w}^{m} \mathbf{U}_{[t: n-m+t-1, t: n-m+t-1]}^{-1}
$$

where $w=1$ or 2 . Ultimately, this process results in the approximation to the solution vector $\mathbf{j}_{n}$ for any frequency $f$ in the range $f_{\min } \leqslant f \leqslant f_{\max }$ given by [5], [7], [8]:

$$
\mathbf{j}_{n}=\mathbf{M}_{n}\left(\sum_{m=0}^{\mathcal{O}} \sigma^{m} \mathbf{M}_{n}^{T} \mathbf{Z}_{m} \mathbf{M}_{n}\right)^{-1}\left(\sum_{m=0}^{\mathcal{O}} \sigma^{m} \mathbf{M}_{n}^{T} \mathbf{v}_{m}\right)
$$

Clearly, Equation 17 can be used to efficiently solve over a wide range of frequencies as it requires the inversion of a matrix of order $n \ll m$ for each frequency value.

\section{A. Implementation}

Determining the optimum size of the approximation order $n$, will result in a more efficient approximation, as the maximum amount of relevant information will be extracted at an expansion point. An approach used in [5], checks whether an $\mathbf{m}_{n+1}$ vector is largely contained in the space $\mathbf{Z}_{0} \mathbf{M}_{n}$. This will occur when the iteration process starts to stagnate as no new useful information will be contained in $\mathbf{m}_{n+1}$ and therefore, it will not be expected to improve the approximation. This approach involves monitoring the projection of:

$$
\mathbf{y}_{n+1}=\mathbf{v}_{n}-\sum_{l=1}^{n} \mathbf{Z}_{l} \mathbf{m}_{n+1-l}
$$

onto the space $\mathbf{Z}_{0} \mathbf{M}_{n}$. To justify the generation of $\mathbf{m}_{n+1}$, we consider how much of $\mathbf{y}_{n+1}$ is contained in this space by forming:

$$
\tilde{\mathbf{y}}_{n+1}=\mathbf{Z}_{0} \mathbf{M}_{n}\left(\mathbf{M}_{n}^{T} \mathbf{Z}_{0} \mathbf{M}_{n}\right)^{-1} \mathbf{M}_{n}^{T} \mathbf{y}_{n+1}
$$

and then check if $C_{n}<=t o l_{c n}$ for some tolerance value $t o l_{c n}$, where

$$
C_{n}=\frac{\left\|\mathbf{y}_{n+1}-\tilde{\mathbf{y}}_{n+1}\right\|_{2}}{\left\|\mathbf{y}_{n+1}\right\|_{2}} .
$$

If the tolerance $t o l_{c n}$ has been met, then $\mathbf{m}_{n+1}$ should not be generated and the MCAWE process terminated, signalling that linear independence has been lost.

\section{Numerical Results}

The first example is a homogeneous square plate of side length $0.02 \mathrm{~m}$, centered at the origin. The plate is illuminated by an plane wave with broadside incidence $\theta_{i n c}=\pi / 2, \phi_{i n c}=$ 0 and polarization $\alpha_{i n c}=\pi / 2$. The plate was discretised using $m=936$ cells and the RCS was computed over a band of frequencies $f=5.0: 35.0 \mathrm{GHz}$ with $1.0 \mathrm{GHz}$ increments for a monostatic setup $\phi_{s c a}=0$. Figure 1 compares the MoM results against the Padé via AWE with adaptive zeta $(n=$ $20)$ and WCAWE $(n=24)$. The Padé via AWE was limited to only 20 moments as the Padé coefficient matrix became singular for any greater value of $n$. Figure 1 shows the RCS versus frequency with one expansion point at $\sigma_{0}=20.0 \mathrm{GHz}$. The increased accuracy of the WCAWE algorithm is clearly evident as it duplicates the reference solution over the band of frequencies $f=8: 32 \mathrm{GHz}$ with a $1 \%$ relative error. While the Pade is limited to a similar error over the band $f=12: 28$ GHz. Figure 2 monitors the linear dependence of the generated 
$\mathbf{m}_{n}$ by displaying $C_{n}$ (Equation 20) versus the iteration number. Termination of the iteration occurs when the prespecified $t o l_{c n}=10^{-4}$ [5] is reached, signalling stagnation in the WCAWE process. Figure 3 illustrates the RCS for varying observation angle $\theta_{s c a}=0: 360$, for an approximation frequency at $f=8 \mathrm{GHz}$.

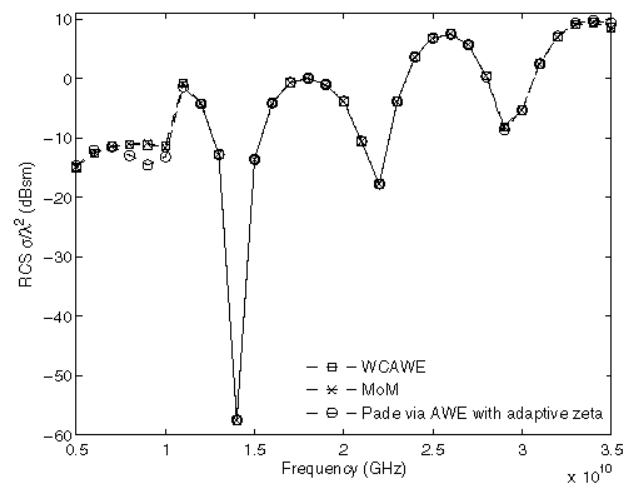

Fig. 1. $\sigma\left(\Phi_{s c a}\right)$ RCS frequency sweep using MoM, Padé via AWE with adaptive zeta, and WCAWE.

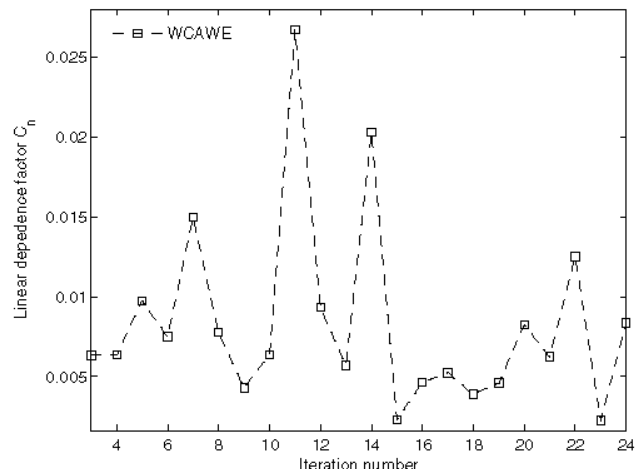

Fig. 2. $C_{n}$ monitoring the linear independence of the generated WCAWE $\mathbf{m}_{n}$

\section{Conclusions}

A fast frequency sweep method for a surface integral equation formulation has been demonstrated using the WCAWE approach. The WCAWE method provides the flexibility needed to efficiently handle the short coming of the AWE with Padé; specifically, the loss of accuracy as $n$ increases due to the explicit moment matching process and the ill-conditioned Padé coefficient matrix. Examples were presented which demonstrate that the WCAWE can produce a numerically stable and robust high order approximation from a single expansion point as compared to the Padé via AWE with adaptive zeta. Finally, an analytical process for monitoring the linear independence of the generated moment vectors has been applied.

\section{Appendix 1. The MCAWE Algorithm}

$\tilde{\mathbf{m}}_{1}=\mathbf{Z}_{0}^{-1} \mathbf{v}_{0}$

$\mathbf{U}_{[1,1]}=\left\|\tilde{\mathbf{m}}_{1}\right\|_{2}$

$\mathbf{m}_{1}=\tilde{\mathbf{m}}_{1} \mathbf{U}_{[1,1]}^{-1}$

for $n=2, \ldots, q$

$$
\begin{aligned}
& \tilde{\mathbf{m}}_{n}=\mathbf{Z}_{0}^{-1}\left(\sum_{m=1}^{n-1}\left(\mathbf{v}_{m} \mathbf{e}_{1}^{T} \mathbf{P}_{\mathrm{U} 1}(n, m) \mathbf{e}_{n-m}\right)\right. \\
& \left.-\mathbf{Z}_{1} \mathbf{m}_{n-1}-\sum_{m=2}^{n-1} \mathbf{Z}_{m} \mathbf{M}_{n-m} \mathbf{P}_{\mathrm{U} 2}(n, m) \mathbf{e}_{n-m}\right) \\
& \text { for } \alpha=1, \ldots, n-1 \\
& \quad \mathbf{U}_{[\alpha, n]}=\mathbf{m}_{\alpha}^{H} \tilde{\mathbf{m}}_{n} \\
& \quad \tilde{\mathbf{m}}_{n}=\tilde{\mathbf{m}}_{n}-\mathbf{U}_{[\alpha, n]} \mathbf{m}_{\alpha} \\
& \text { end } \alpha \\
& \mathbf{U}_{[n, n]}=\left\|\tilde{\mathbf{m}}_{n}\right\|_{2} \\
& \mathbf{m}_{n}=\tilde{\mathbf{m}}_{n} \mathbf{U}_{[n, n]}^{-1}
\end{aligned}
$$$$
\text { end } n \text {. }
$$

\section{REFERENCES}

[1] A. F. Peterson, S. L. Ray and R. Mittra, "Computational Methods for Electromagnetics," IEEE Press, Piscataway, New Jersey, USA, 1998.

[2] S. M. Rao, D. R. Wilton, and A. W. Glisson, "Electromagnetic scattering by surfaces of arbitrary shape," IEEE Trans. Antennas Propagat., vol. 30, no. 3, pp. 409-418, 1982.

[3] B. Fasenfest, J. D. Rockway, N. J. Champagne, and R. M. Sharpe, "A generalized fast frequency sweep algorithm for coupled circuitEM simulations," IEEE Antennas and Propagation Society International Symposium, vol. 4, pp. 3944-3947, June 2004.

[4] K. Gallivan, E. Grimme, and P. Van Dooren, "Padé Approximation of large-scale dynamic systems with Lanczos methods," Proc. 33rd IEEE Conf. on Decision and Control, 1994.

[5] R. D. Slone, "Fast frequency sweep model order reduction of polynomial matrix equations resulting from finite element discretizations," $\mathrm{Ph} . \mathrm{D}$. Dissertation, Ohio State University, Columbus, OH, 2002.

[6] C. J. Reddy, M. D. Deshpande, C. R. Cockrell and F. B. Beck, "Fast RCS computation over a frequency band using method of moments in conjunction with asymptotic waveform evaluation technique," IEEE Trans. Antennas Propagat., vol.46, iss.8, pp. 1229-1233, Aug 1998.

[7] J. X. Wan and C. H. Liang, "Rapid solutions of scattering from microstrip antennas using well-conditioned asymptotic waveform evaluation," Progress In Electromagnetics Research, PIER 49, pp. 39-52, 2004.

[8] R. D. Slone, R. Lee, and J. F. Lee, "Well-conditioned asymptotic waveform evaluation for finite elements," IEEE Trans. Antennas Propagat., vol. 51, pp. 2442-2447, 2003.

Fig. 3. $\sigma\left(\Phi_{s c a}\right)$ RCS for a converged frequency $f=8 \mathrm{GHz}$. 